\title{
Vulnerability of Ultra-large Urban Traffic System based on Complex Network and Topological Potential
}

\author{
Tao Sun ${ }^{1}$, Changlin Jiang ${ }^{2}$ and Anning $\mathrm{Ni}^{1, *}$ \\ ${ }^{1}$ School of Naval Architecture, Ocean and Civil Engineering, Shanghai Jiao Tong University, Shanghai 200240, China \\ ${ }^{2}$ College of Engineering, Carnegie Mellon University, Pittsburgh, PA 15213, United States
}

Received 27 August 2018; Accepted 15 November 2018

\begin{abstract}
Vulnerability of traffic network is a key problem of the traffic industry due to the intensifying traffic jams and increasing traffic accident rate. However, existing analyses on vulnerability of traffic network only focus on statistical characteristics of complex network. These works inevitably have some shortages, such as single network structure, low conformity between evaluation and actual monitoring results, and failure to focus on important traffic facilities (e.g., large bridges and tunnels). To solve problems in the existing evaluation model of traffic network vulnerability, a comprehensive evaluation model that integrated complex network and topological potential was proposed in this study. First, the urban road network was abstracted into a complex network topology based on complex network theory. Network attributes, which could influence vulnerability of traffic network, were extracted. Second, the influence range of traffic was analyzed on the basis of the network radiation model and congestion propagation theory. Finally, vulnerability indexes of traffic network were defined. Vulnerability of bridges and tunnels in the entire traffic network were assessed from physical attributes of roads, traffic conditions, and topological characteristics of traffic network. Results show that (1) abstracting the traffic network into a complex network is a high-efficiency processing method, whereas the combination of the network radiation model and congestion propaganda theory overcomes not only dependence of the gravity model on adjustable parameters during traffic assignment but also difference brought by parameters. Thus, the traffic assignment is objective and the results are accurate. (2) Determining the value of influencing factor is important. "Physical attributes of roads," "traffic attributes," and "structure attributes of traffic network" can directly affect the value of influencing factor accurately. The evaluation becomes accurate when a large number of influencing attributes are considered. The comprehensive evaluation model integrating complex network and topological potential can evaluate vulnerability of the traffic network effectively. The model provides a new perspective and method to study vulnerability of the traffic network.
\end{abstract}

Keywords: Vulnerability of road network, Congestion propagation, Dynamic traffic assignment, Large tunnel and bridge

\section{Introduction}

The urban traffic system expands as a response to the continuous expansion of urban size accompanied with mutual interactions of parts and abundant influencing factors Thus, urban traffic system is a typical and open complex network. Moreover, the frequent traffic jams and accidents brought by increasing traffic demands and road construction can influence the overall performance of urban road network and may even cause large-scaled traffic paralysis in downtown areas due to the spreading of traffic congestion in rush hours. Many large potential risks are present in the running of trunk road network in cities, such as fluctuation of traffic demands, earthquake, heavy snow and freezing, strong wind, flood, large-scaled activities, and terrorist attacks. These risks can decrease the traffic capacity and may even cause paralysis of the entire network [1]. Many crossing-river channels, including crossing-river tunnels and bridges, have been constructed along seas and rivers in urban traffic network to meet daily traffic demands of

\footnotetext{
*E-mail address: nianning@situ.edu.cn

ISSN: $1791-2377$ @ 2018 Eastern Macedonia and Thrace Institute of Technology. All rights reserved.

doi:10.25103/jestr.115.06
}

residents. Crossing-river bridges and tunnels are mainly highways and express ways with at least two-way four lanes. They can reduce environmental pollution and oil consumption, shorten travel time, and connect the traffic network. For example, many roads in megacities (e.g., Shanghai) in China have been saturated with the increase in number of cars and population although two banks of the Huangpu River have been connected [2]. In particular, the capacity to absorb new road networks weakens gradually during the morning and evening rush hours with serious congestion of the entire trunk network [3]. Developing functions of large bridges and tunnels is difficult during weather disasters and may even cause traffic accidents and large-scaled reach of traffic congestion. Therefore, large bridges and tunnels have encountered bottlenecks in guiding traffic flows in the road network [4]. Thus, studying the importance of crossing-river tunnels and bridges in the entire urban traffic network and discussing the influences of similar crossing-river facilities in the entire urban traffic network under faults are imperative.

Existing studies on vulnerability of traffic network mainly focus on public transportation and subway networks $[5,6,7,8]$. On the contrary, only a few studies on urban trunk network have been reported. Vulnerability of traffic network is mainly studied by complex network theory and 
simulation assessment. The complex network emphasizes on the system structure and analyzes functions and statistical characteristics of systems from the structural perspective. However, the structural information overlooks traffic characteristics and other problems. Simulation assessment evaluates vulnerability of the road network by simulating the microscopic or macroscopic scenes of traffic network by using simulation software. Nevertheless, setting of simulation parameters influences vulnerability assessment considerably, and travelers' knowledge on road information can also influence the consequence evidently [9]. Studies on vulnerability of traffic network pay small attention to mutual influences of factors. According to existing research achievements on vulnerability of traffic network, most results are inaccurate and the cooperation of different influencing factors is barely concerned.

In consideration of the increasingly complicated new traffic conditions, research method of complex systems has gained overall understanding of the traffic system and provided theoretical references for solving urban traffic problems. In this study, complex network theory, network radiation model, and congestion propagation theory were combined. A calculation method of vulnerability of traffic network was defined using structural characteristics of road network topology, physical attributes of roads, and traffic characteristics as the reasoning evidence. The proposed method is conducive to increase evaluation accuracy of vulnerability of the traffic network. This method is important in maintaining normal running of regional traffic, managing large traffic flows, increasing the overall efficiency of the road network, and improving the ability to cope with emergency events.

\section{State of the art}

Studies on vulnerability of traffic road network began in the 1980s and shifted attentions from vulnerability of road network caused by natural disasters (e.g., earthquake, mudslide, flooding, and landslide) to the vulnerability caused by traffic accidents and road damages. An increasing number of academic studies and conference reports on vulnerability of traffic network are available due to its increasing importance in the traffic field. Berdica [10] proposed the first definition of vulnerability of road network. He believed that vulnerability of the traffic network is a senstivity coefficient to events and can decrease the extreme of service level of the road network. Descriptions of vulnerability can generally be divided into two types. First, some scholars pointed out that vulnerability of road network is related to failure of some components in the network and emphasizes on corresponding consequences. For example, M D'Este and Taylor [11] reported that vulnerability considers consequence of disasters only. In other words, failure severity of some compnents decreases the accessibility to some nodes (the accesibility index is the vulnerability of nodes). Second, other scholars concluded that vulnerability of road network is closely related to risks and that the probability of link failure should be considered except for consequences of failure. Jenelius and Mattsson [12] argued that the concept of vulnerability should be divided into two parts: (1) probability of occurrence of risk events and (2) consequences of events at a specific site. However, no unified standards and no reliable theoretical model of urban traffic system have been formed yet.
Traffic network is a complex network system integrating urban planning and roads. Complex network theory is widely used in traffic network and provides another important measurement method of links in the worldwide public transport network $[13,14]$. With respect to the size of the largest link subset, vulnerability of network is more sensitive to betweenness centrality than node degree. Çolak and Luş [15] defined vulnerability by the breaking speed of the system and obtained same conclusions. Complex network theory proposes many vulnerability indexes of network based on the topological structure of complex network (Table 1), including degree, degree distribution, shortest distance, shortest average distance, clustering coefficient, and betweenness [16]. Sienkiewicz and Holyst [5] analyzed topological structural characteristics of the public transport networks in 21 cities of Poland. Subsequently, they further analyzed clustering coefficient, matching attribute, and betweenness of public transport network in cities of Poland. However, they ignored actual attributes and traffic characteristics of roads.

Although theoretical analysis and numerical applications deepen understanding on the simultaneous effect of supply and demand on vulnerability, most analyses on vulnerability of transport network concentrate on degeneration of infractures and major accidents or characteristics and some economic features of the traffic network, especially in road network and individual driving travel [17]. Husdal [18] disclosed that vulnerability of network is related to network flow and attributes related to environmental factors despite of the network structure. Nevertheless, the evaluation model involves many subjective factors, thereby resulting in poor evaluation accuracy. Zhang and Wang [6] analyzed networking characteristics of subway networks in Shanghai, Beijing, and Guangzhou. They also investigated vulnerability of subway network by two malicious attacking methods based on connectivity and functional vulnerabilities. They explored an improved topological structure of subway network as well. Rodríguez-Núñez and García-Palomares [7] measured criticality and vulnerability of public transport network by complex network indexes. They analyzed the increased traveling time caused by choosing the optimal route by using the actual network flow distribution. However, the public transport and subway networks have inadequate complexity. The authors used only statistical reasoning methods, such as complex network and hidden Markov chain, which cannot provide a comprehensive evaluation process and involve inadequate structural information. $\mathrm{Li}$ and Guo [8] described the coupling structure between the public transport and subway networks by analyzing the tolerance of traffic network under different attacking modes. Although the coupling structure is complicated, the complexity is still low and structural information is inadequate to capture single characteristic or characteristics of one aspect of the public transport network. However, the traffic network is a complicated multi-factor structure and should be studied in consideration of multiple factors.

In summary, existing studies on vulnerability of traffic network concentrate on network and statistical characteristics of complex network. Only a few studies have evaluated vulnerability of road network by combining traffic characteristics and physical attributes of roads. Here, the topological potential field that can combine different characteritics was introduced to evaluate vulnerability integrating characteristics of traffic network, traffic characteristics, and physical attributes of roads. Inspired by physical field, the theory of topological potential field is an 
important component of field theory. Topological potential is defined as the differential positions of each node in the topology. It is determined by the topology structure of node environment and is defined by the form of Gaussian function [20]. Topological potential energy covers rich structural information of the traffic network and structural information of roads. It offers an ideal solution to inadequate structural information in the traditional Laplacian matrix. Thus, a new method for vulnerability evaluation based on topological potential and complex network was proposed in this study.

Table 1. Complex network theory conceptions [19]

\begin{tabular}{|c|c|c|c|}
\hline $\begin{array}{c}\text { Network } \\
\text { characteristics }\end{array}$ & Concept description & Formula & Meaning of Parameters \\
\hline Degree & $\begin{array}{l}\text { It refers to the number of edges associating the node with other } \\
\text { connected nodes. Network degree distribution refers to nodes } \\
\text { with a probability or proportion that is exactly k expressed with } \\
\text { distribution function } \mathrm{p}(\mathrm{k}) \text {. }\end{array}$ & $k_{i}=\sum_{j, j \neq i} a_{i j}$ & $\begin{array}{l}a_{i j} \text {-relative value in adjacency } \\
\text { matrix } \\
\mathrm{N} \text {-number of nodes in the entire } \\
\text { network }\end{array}$ \\
\hline $\begin{array}{l}\text { Average path } \\
\text { length }\end{array}$ & $\begin{array}{l}\text { It refers to average distance between all pairs of nodes. For the } \\
\text { road network model, it is an indicator of road network } \\
\text { connectivity and convenience of travel. }\end{array}$ & $L=\sum_{\mathrm{i}=1}^{N} \sum_{j=1}^{N} \frac{2 L_{i j}}{N(N-1)}$ & $\begin{array}{l}L_{i j} \quad \text {-shortest path length } \\
\text { between any two nodes } \\
\mathrm{N} \text {-number of nodes in the entire } \\
\text { network }\end{array}$ \\
\hline $\begin{array}{l}\text { Clustering } \\
\text { coefficient }\end{array}$ & $\begin{array}{l}\text { A large network clustering coefficient indicates high connection } \\
\text { tightness between the intersection and nearby intersections and } \\
\text { high density of the road network. High connection tightness is } \\
\text { convenient for traffic travel. }\end{array}$ & $C_{i}=\frac{2 e_{i}}{k_{i}\left(k_{i}-1\right)}\left(k_{i} \geq 2\right)$ & $\begin{array}{l}e_{i} \text {-actual number of edges } \\
\text { connected with node } \mathrm{i}\end{array}$ \\
\hline Betweenness & $\begin{array}{l}\text { It indicates the number proportion of the shortest path through } \\
\text { the node (edge) in the network. Betweenness is a key indicator } \\
\text { of discovering and protecting key nodes (edges). }\end{array}$ & $B_{i}=\sum_{k, j}$ & $\begin{array}{l}D_{\mathrm{k} j}(i) \text {-number of the shortest } \\
\text { path through the node } \mathrm{i} \\
D_{k j} \text {-number of the shortest path } \\
\text { between nodes } \mathrm{k} \text { and } \mathrm{j}\end{array}$ \\
\hline $\begin{array}{l}\text { Network } \\
\text { efficiency }\end{array}$ & $\begin{array}{l}\text { It shows the effectiveness of composite network after removing } \\
\text { certain nodes. }\end{array}$ & $E(G)_{g l o b}=\frac{\sum_{\forall i, j i \neq j} \frac{1}{d_{i j}}}{N(N-1)}$ & $\begin{array}{l}d_{i j} \text { - shortest distance between } \\
\text { node } \mathrm{i} \text { and node } \mathrm{j} \\
\mathrm{N} \text {-number of nodes in the entire } \\
\text { network }\end{array}$ \\
\hline $\begin{array}{l}\text { Eigenvector } \\
\text { centrality }\end{array}$ & \multicolumn{3}{|c|}{$\begin{array}{l}\text { It is a measure of the influence of a node in a network. It assigns relative scores to all nodes in the network depending on the } \\
\text { concept that connections to high-scoring nodes contribute more to the score of the node in question than equal connections to low- } \\
\text { scoring nodes. }\end{array}$} \\
\hline
\end{tabular}

The remainder of the study is organized as follows. Section 3 proposes the vulnerability evaluation method for traffic network based on the complex network model, network radiation model, and congestion propagation theory and the vulnerability indexes of the traffic network. Section 4 presents a case study of the trunk network within the outer ring of Shanghai. The vulnerability of 4 crossing-river bridges and 13 crossing-river tunnels in Shanghai is evaluated with the traffic test data of Shanghai Transportation Commission. Section 5 elaborates the conclusions.

\section{Methodology}

\subsection{Topological structure of the traffic network}

The structural diagram of traffic network is composed of a group of nodes and links. It allows flow of information or objects in an n-dimensional space. The traffic network $(G)$ can be described as $G=(N, A)$, where $N=\left\{\mathrm{n}_{i} \mid i=1,2,3 \mathrm{~L} n\right\}$ is the node set, $\mathrm{n}$ is the total number of nodes, and $A=\left\{a_{i j}=\left(v_{i}, v_{j}\right) \mid v_{i}, v_{j} \subset N\right\}$ is the link set. Moreover, $|E|=m$. If the link $a_{i j}$ exists between nodes $\mathrm{i}$ and $\mathrm{j}$, then $a_{i j}=1$; otherwise, $a_{i j}=0$.

Two important structural attributes of complex network, which are used in many other evaluation systems, are utilized in the present study. An important step is to measure properties of a small typical neighborhood group by the clustering coefficient $C_{i}$. Another step is to measure the typical distance between two nodes by the path length $L_{i j}$, which is introduced by Watts and Strogatz [21]. Clustering considers the number of direct links between adjacent nodes $\left(k_{i}\right)$. The clustering coefficient of nodes is equal to $k_{i}$ divided by the number of possible links between them. Derrible and Kennedy [22] reported that the maximum number of links in a plane network is $3 k_{i}-1$, where $k_{i} \geq 3$. Therefore, the clustering coefficient $C_{i}$ of node $\mathrm{i}$ can be expressed as

$$
\begin{aligned}
C_{i} & =\frac{k_{i}}{3\left(k_{i}-1\right)}\left(k_{i} \geq 3\right) \\
k_{i} & =\sum_{j, j \neq i} a_{i j}
\end{aligned}
$$

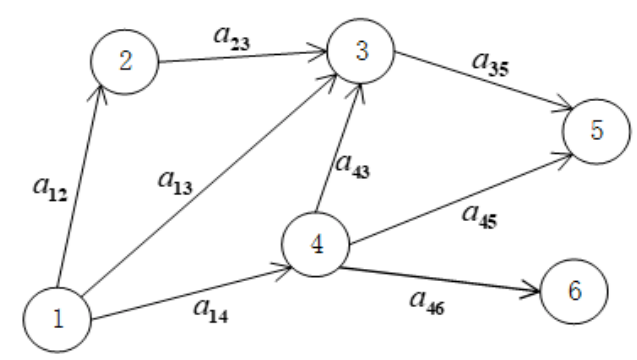

Fig. 1. Traffic network (G)

A high clustering coefficient reflects high connection tightness between nodes, which is convenient for traffic travel.

On this basis, the network efficiency can be gained. Network efficiency is an index that measures flowability or mobility in the traffic network. The characteristic path length 
$\left(L_{i j}\right)$ is used to measure the network efficiency. The reason is that a low value of $L_{i j}$ reflects high moving speed between any pair of nodes [23]. The communication efficiency $\eta(G)$ between nodes $\mathrm{i}$ and $\mathrm{j}$ can be defined as the reciprocal of characteristic path length $L_{i j}=+\infty$.

When no connecting path exists between nodes $\mathrm{i}$ and $\mathrm{j}$. The global network efficiency $\eta(G)$ reflects the overall capability of the network, where G generally represents the traffic network. $\eta(G)$ is defined as follows [24]:

$$
\eta(G)=\frac{1}{|N|(|N|-1)} \sum_{i, j \subset N ; i \neq j} \frac{1}{L_{i j}}
$$

\subsection{Topological potential}

Nodes are connected by links and influence mutually rather than isolated in the topological structure of a complex network. Therefore, the theory of topological potential field is introduced into the complex network to describe the relationships of nodes and disclose the basic characteristics of potential importance distribution [20,25].

The theory of topological potential field posits that the topological potential of any node in a given traffic network $G=(N, A)$ can be defined as

$$
\varphi\left(v_{i}\right)=\sum_{i=0}^{k}\left[m_{1}\left(v_{i}\right)+\mathrm{L}+m_{s}\left(v_{l}\right)\right] \times e^{-\left(L_{i l} / \sigma\right)^{2}}
$$

where $\varphi\left(v_{i}\right)$ is the topological potential of node $v_{i}(1 \leq i \leq n)$. Node $v_{l}$ is the node within the influence scope of node $v_{i} . \mathrm{k}$ is the total number of nodes in the influence scope of $v_{i}$, and $1 \leq k \leq n-1$ and $1 \leq l \leq k$ exist. $L_{i l}$ is the shortest path between nodes $v_{l}$ and $v_{i} . m_{s}\left(v_{l}\right)$ is the quality or inherent attribute of the node $v_{l}$ and may have different physical importance. $\sigma$ is the influence scope of one influencing factor over the node $v_{i}$.

The influencing factor $\sigma$ is the influence scope of node $v_{i}$. If $\sigma \rightarrow 0$, then no mutual influence is exerted among different nodes. On the contrary, the mutual influence of nodes is very strong and all nodes even influence mutually if $\sigma \rightarrow \infty$. The value of $\sigma$ influences the accuracy of topological potential results. $\sigma$ is optimized and selected by calculating the topological potential entropy [26]. The topological potential entropy $H$ is defined as

$$
H=-\sum_{i=1}^{n} \frac{\varphi\left(v_{i}\right)}{Z} \log \frac{\varphi\left(v_{i}\right)}{Z}
$$

where $\mathrm{n}$ the total number of nodes and $Z=\sum_{i=1}^{n} \varphi\left(v_{i}\right)$ is a normalized factor. The topological potential distribution is the mostly uneven, and the uncertainty is the minimum under the minimum topological potential entropy. At this moment, the value of influencing factor is the desired optimal value.

\subsection{Radiation model of the topological network}

Among the traffic assignment and travel demand models, gravity model is the most important $[27,28]$. Gravity model originated from Newton's law of gravitation. Such model is extensively used but depends on adjustable parameters. These parameters vary in different regions and may disagree with known analysis [29]. The gravity model has evident limitations, and its validity is still controversial. Compared with the survey data, the gravity model fails to estimate travel attraction accurately [30, 31]. Simini and González [29] introduced a radiation model to predict communication cpacity between the long-term migration mode and different regions. The observed migration mode agrees well with the transmission and moving modes. Without parameter characteristics, this model can be used in fields that lack prior moving measurement and considerably increases the prediction accuracy of most phenomena influenced by moving and transmission processes.

The radiation model of topological network is defined on the basis of the gravity and radiation models. In the present study, a real road network is dualized. The actual roads are nodes in the dual network and nodes are links in the dual network. The topological potentials of nodes $\mathrm{i}$ and $\mathrm{j}\left(\varphi\left(v_{i}\right)\right.$ and $\left.\varphi\left(v_{j}\right)\right)$ are considered. The node degree $\left(k_{i}\right)$ in the actual network corresponding to nodes $\mathrm{i}$ and $\mathrm{j}$ in the dual network is considered. The average traffic volume from node $i$ to node $j$, which is predicted by the radiation model of topological network, can be defined as $T_{i j}$ :

$$
T_{i j}=T_{i} \frac{\varphi\left(v_{i}\right) \varphi\left(v_{j}\right)}{\left(\varphi\left(v_{i}\right)+\sum_{i=1}^{h} k_{i}\right)\left(\varphi\left(v_{i}\right)+\varphi\left(v_{j}\right)+\sum_{i=1}^{h} k_{i}\right)}
$$

where $h$ is the number of links between nodes $\mathrm{i}$ and $\mathrm{j}$. The absorbed traffic $\left(T_{i j}\right)$ considers inherent attributes of roads and structural attributes in the topological network.

\subsection{Vulnerability evaluation of bridges and tunnels}

Many researchers [32], including researchers engaged in traffic network [33], defined change rate of network efficiency as the vulnerability of network. This vulnerability refers to the reduction in global safety of the network caused by interruption of links in the network. The definition of vulnerability only considers that the change in topological network efficiency is incomplete and inaccurate. Therefore, the change rate of global efficiency in the present study is called the topological vulnerability $\left(V_{T}\right)$ and is used as one of evaluation indexes of vulnerability.

$V_{T}=\frac{\eta(G)-\eta\left(G^{*}\right)}{\eta(G)}$

where $G^{*}$ is the new network produced by the original traffic network $\mathrm{G}$ when the fault links are eliminated.

Change rate of the traffic network potential is another evaluation index of vulnerability. In accordance with the definition of change rate of the global efficiency, the change rate of topological potential can be defined as

$$
V_{Z}=\frac{Z-Z^{*}}{Z}
$$

where $Z$ and $Z^{*}$ are the sum of topological potentials of all nodes in the traffic network, which are corresponding to the traffic networks $\mathrm{G}$ and $G^{*}$.

Leamer [34] (Sections 4.4-4.6) proposed the Bayesian averaging of Bayesian estimates and introduced the basic equation. The Bayesian averaging of classical estimates was proposed by Raftery [35] and then modified by Doppelhofer 
and Miller [36]. The Bayesian averaging method has been studied and applied extensively [37-40]. Inspired by the Bayes mean, the vulnerability is defined as follows:

$V=\frac{m V_{T}+n V_{Z}}{n+m}$

where $\mathrm{n}$ and $\mathrm{m}$ are total numbers of nodes and links in the network, respectively.

\section{Case study}

\subsection{Data source and topological modeling}

In the case study, all data were provided by Shanghai Transportation Commission and covered road conditions, information of crossing-river bridges and tunnels, road capacity, intersection information, road network structure, and traffic flows in different road sections and crossing from April 2016 to May 2016. Among them, traffic flow was used as two-way statistical data and the unit in this study was "passenger car unit."

The topological structure of trunk network in Shanghai (including 13 large crossing-river tunnels and 4 crossingriver bridges, which are key research objects in the road network) was constructed in accordance with the characteristics of trunk network and crossing-river bridges and tunnels (Fig. 2). The intersections in the road network and road sections between two intersections were abstracted as nodes and edges of two nodes, respectively. Characteristics of a complex network and actual road network (Table 1) were used as influencing factors of comprehensive vulnerability evaluation of traffic network.

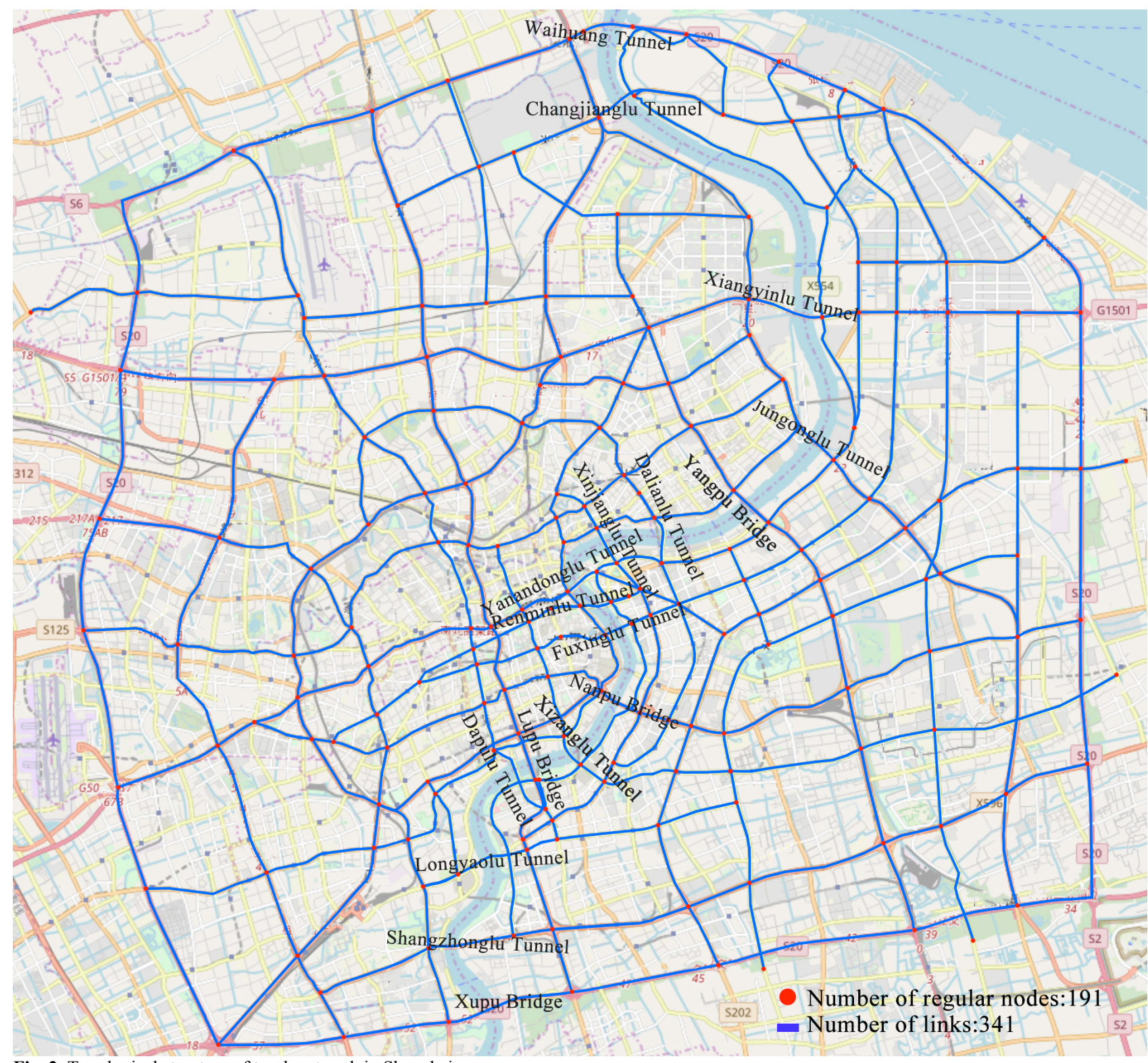

Fig. 2. Topological structure of trunk network in Shanghai

\subsection{Vulnerability analysis of large bridges and tunnels in Shanghai}

4.2.1 Calculation of topological potentials and value of influencing factor

In the present study, topological attributes $\left(k_{i}, L_{S i l}, C_{i}\right.$ and $\left.m_{S N d}\right)$ of the traffic network and physical attributes $\left(m_{T r v}\right.$, $m_{T r c}$ and $m_{L a}$ ) of the road network were chosen as attributes 
for comprehensive evaluation. Table 2 introduces these attributes.

Table 2. Variable

\begin{tabular}{|c|c|c|}
\hline \multicolumn{2}{|c|}{ Variable } & Variable description \\
\hline \multicolumn{2}{|c|}{$k_{i}$} & Node degree \\
\hline \multicolumn{2}{|c|}{$L_{i j}$} & Segment length \\
\hline \multicolumn{2}{|c|}{$L_{S i l}$} & Shortest path length between node $i$ and $l$ \\
\hline \multirow{5}{*}{$m_{s}\left(v_{l}\right)$} & $T_{v}$ & $\begin{array}{l}\text { Average daily peak hour traffic volume from April } \\
2016 \text { to May } 2016\end{array}$ \\
\hline & $T_{c}$ & Traffic capacity of each lane \\
\hline & $C_{i}$ & Clustering coefficient \\
\hline & $k_{S i j}$ & $\begin{array}{l}\text { Degree of the segment node after the dual of the } \\
\text { road network }\end{array}$ \\
\hline & $m_{L a}$ & Number of lanes corresponding to a segment \\
\hline
\end{tabular}

The topological network in Fig. 1 was converted to a dual network. Nodes in the original traffic network were converted into links, and links in the original traffic networks were changed into nodes. Topological potential of nodes in the dual network was calculated in accordance with Eq. (4). In this evaluation method, finding the value of the influencing factor $(\sigma)$, which represents the influence range of nodes, is important. The functional image of the topological potential entropy is shown in Fig. 3, where the minimum of $\sigma$ is 0.1200 . The minimum of the influencing factor in this case study is determined to be 0.1200 .

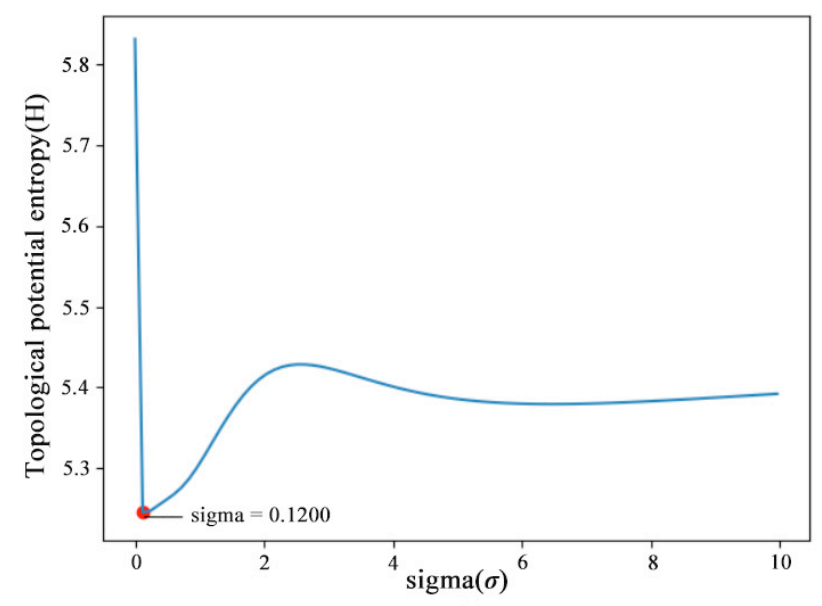

Fig. 3. Influencing factor

\subsubsection{Calculation of reach}

Bridges or tunnels may be closed for traffic during accidents or disaster attacks, which surely influence the surrounding traffic roads. To study vulnerability of large bridges and tunnels, roads that are connected with each bridge or tunnel were attacked successively and traffic assignment was performed. Each flow assignment was accomplished in accordance with Eq. (6), which was close to practical situations. The links would fail when $m_{T r v} \geq m_{L a} \times m_{T r c}$, and failed links were deleted from the traffic network. In accordance with congestion propagation theory, links that meet $m_{T r v} \geq m_{L a} \times m_{T r c}$ in all roads were deleted after each assignment, and traffic was redistributed until no failed links are found in the road network.

From the analysis of 13 large crossing-river tunnels and 4 crossing-river bridges in Shanghai, they were assumed to be damaged upon attacks. Fig. 4 shows the influence range of each tunnel and bridge. Different traffic facilities are expressed by different colors.
Depending on connections between nodes and links in the topological structure of the trunk network in Shanghai, the global efficiency of the road network $\left(\eta\left(G^{*}\right)\right)$ after failed links was deleted using Eq. (3). Similarly, the corresponding $Z^{*}$ can be calculated. Table 3 lists the results of different facilities.

Table 3. Network efficiency and potential entropy after failure

\begin{tabular}{l|c|c}
\hline \multicolumn{1}{c|}{ Traffic facilities } & $\eta\left(G^{*}\right)$ & $Z^{*}$ \\
\hline Original & 0.205 & 37002054.97 \\
Nanpu Bridge & 0.168 & 35268054 \\
Waihuan Tunnel & 0.181 & 35888005 \\
Shangzhonglu Tunnel & 0.191 & 34002004.90 \\
Yangpu Bridge & 0.188 & 35002004 \\
Xupu Bridge & 0.185 & 36068054 \\
ChangjiangluTunnel & 0.189 & 34822007 \\
Yan'andonglu Tunnel & 0.186 & 35990000 \\
Xinjianlu Tunnel & 0.187 & 35902999 \\
Lupu Bridge & 0.190 & 35002005 \\
Xiangyinlu Tunnel & 0.194 & 34003000 \\
Jungonglu Tunnel & 0.186 & 36689904 \\
Fuxinglu Tunnel & 0.192 & 35602000 \\
Dalianlu Tunnel & 0.193 & 35322078 \\
Dapulu Tunnel & 0.192 & 36999000 \\
Renminlu Tunnel & 0.196 & 35942265 \\
Longyao Tunnel & 0.195 & 36572116 \\
Xizanglu Tunnel & 0.197 & 36992066 \\
\hline
\end{tabular}

\subsubsection{Vulnerability analysis}

In accordance with connections between nodes and links in the topological structure of the trunk network in Shanghai, the global efficiency of the current road network can be calculated by Eq. (3) as $\eta(G)=0.205$.

\section{Conclusions}

A comprehensive evaluation model based on complex network, network radiation model, and congestion propagation theory was proposed to deal with the intensifying traffic congestion and increasing traffic accidents. The proposed model was used to solve time and spatial diffusion brought by saturation of abundant road sections and reduction in traffic capacity of the entire traffic network caused by potential risk events. This model could evaluate vulnerability of large urban traffic network accurately. The model was also used to study vulnerability of Shanghai road network covering 13 crossing-river tunnels and 4 crossing-river bridges within the outer ring of Shanghai. From the results, the conclusions were obtained as follows:

(1) The combination of network radiation model and congestion propagation theory overcomes shortages of the gravity model during traffic distribution, such as high dependence on adjustable parameters, difference brought by parameters, and inconsistent analyses. The topological potential also combines the physical attributes of roads and network structural attributes well in accordance with attributes of the complex network. As a result, the reliability of the evaluation results increases. The number of evaluation factors can be determined depending on practical situations.

(2) Determining the influencing factor that symbolizes the reach of nodes is imperative. "Physical attributes of roads," "traffic attributes," and "structural attributes of the traffic network" can influence the value accuracy of the 
influencing factor. The evaluation becomes accurate when a large number of influencing factors are considered.

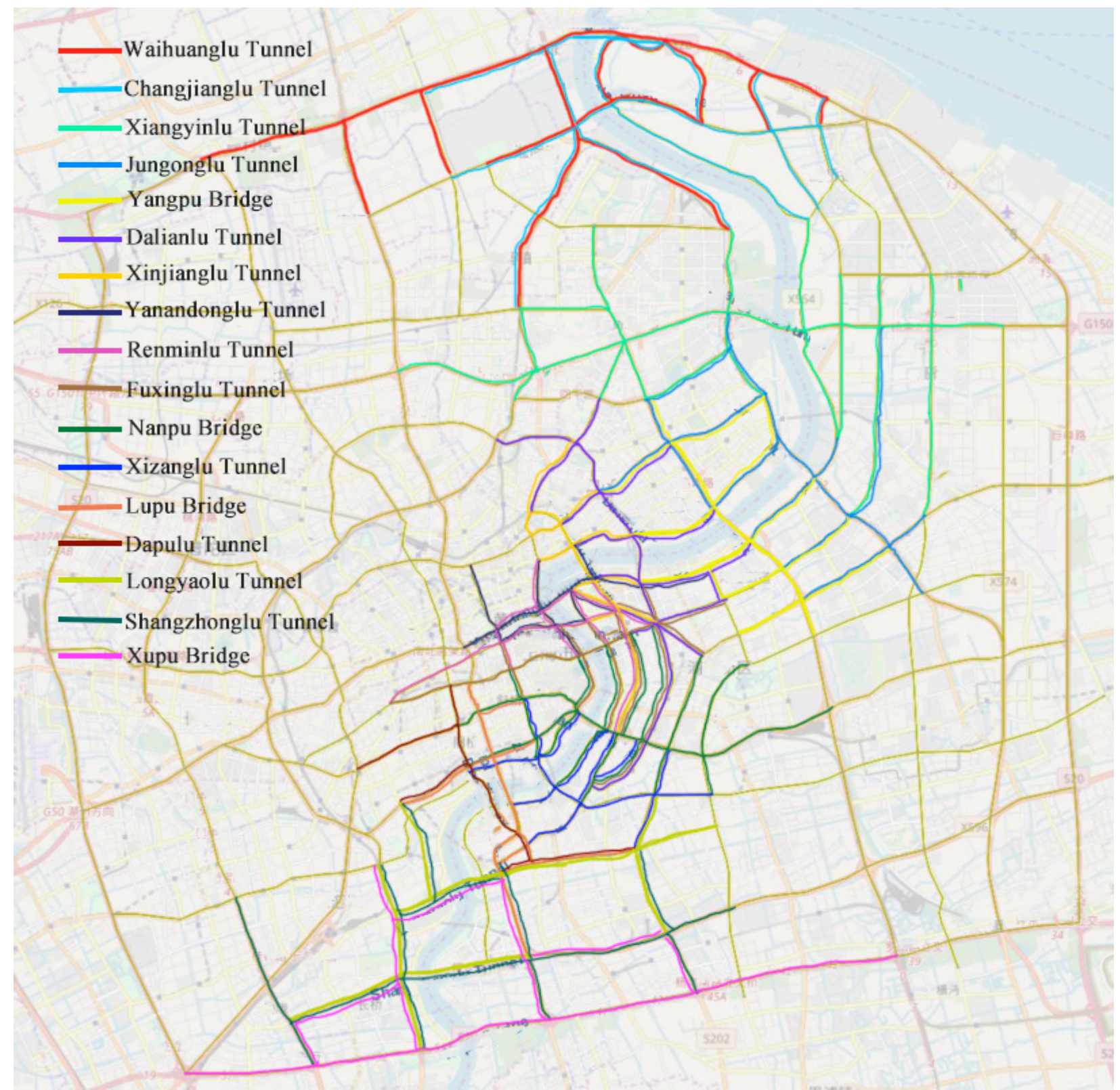

Fig. 4. Reaches of different bridges and tunnel

Table 4. Analysis results of vulnerability of Shanghai Bridge and Tunnel

\begin{tabular}{|c|c|c|c|c|c|c|}
\hline Cross river facilities & $\eta\left(G^{*}\right)$ & $Z^{*}$ & $V_{T}$ & $V_{Z}$ & Vulnerability $V$ & Ranking \\
\hline Original & 0.205 & 37002054.97 & & & & \\
\hline Nanpu Bridge & 0.168 & 35268054 & 0.180487805 & 0.046862288 & $13.25 \%$ & 1 \\
\hline Waihuan Tunnel & 0.181 & 35888005 & 0.117073171 & 0.030107786 & $8.59 \%$ & 2 \\
\hline Shangzhonglu Tunnel & 0.191 & 34002004.90 & 0.068292683 & 0.081077931 & $7.29 \%$ & 3 \\
\hline Yangpu Bridge & 0.188 & 35002004 & 0.082926829 & 0.05405243 & $7.26 \%$ & 4 \\
\hline Xupu Bridge & 0.185 & 36068054 & 0.097560976 & 0.025241868 & $7.16 \%$ & 5 \\
\hline ChangjiangluTunnel & 0.189 & 34822007 & 0.07804878 & 0.058916943 & $7.12 \%$ & 6 \\
\hline Yan'andonglu Tunnel & 0.186 & 35990000 & 0.092682927 & 0.027351318 & $6.92 \%$ & 7 \\
\hline Xinjianlu Tunnel & 0.187 & 35902999 & 0.087804878 & 0.029702566 & $6.69 \%$ & 8 \\
\hline Lupu Bridge & 0.190 & 35002005 & 0.073170732 & 0.054052403 & $6.63 \%$ & 9 \\
\hline Xiangyinlu Tunnel & 0.194 & 34003000 & 0.053658537 & 0.081051038 & $6.35 \%$ & 10 \\
\hline Jungonglu Tunnel & 0.186 & 36689904 & 0.092682927 & 0.008436044 & $6.24 \%$ & 11 \\
\hline Fuxinglu Tunnel & 0.192 & 35602000 & 0.063414634 & 0.037837222 & $5.42 \%$ & 12 \\
\hline Dalianlu Tunnel & 0.193 & 35322078 & 0.058536585 & 0.045402261 & $5.38 \%$ & 13 \\
\hline Dapulu Tunnel & 0.192 & 36999000 & 0.063414634 & $8.25622 \mathrm{E}-05$ & $4.07 \%$ & 14 \\
\hline Renminlu Tunnel & 0.196 & 35942265 & 0.043902439 & 0.028641381 & $3.84 \%$ & 15 \\
\hline Longyao Tunnel & 0.195 & 36572116 & 0.048780488 & 0.011619327 & $3.54 \%$ & 16 \\
\hline Xizanglu Tunnel & 0.197 & 36992066 & 0.03902439 & 0.000269957 & $2.51 \%$ & 17 \\
\hline
\end{tabular}


(3) The proposed model is used to evaluate vulnerability of Shanghai road network covering 13 crossing-river tunnels and 4 crossing-river bridges within the outer ring. The results show that the Nanpu Bridge is very vulnerable to the trunk network in Shanghai. It may decrease the entire traffic network efficiency considerably during accidents, thereby causing large-scaled influences. The Waihuanlu Tunnel, Shangzhonglu Tunnel, Yangpu Bridge, and Xupu Bridge play an important role in the global network efficiency, whereas the Xizanglu tunnel only slightly influences the entire network.

The proposed model not only can be used to evaluate vulnerability of large urban traffic networks but also is applicable to other fields, such as aviation network systems or other systems with complex network attributes. This study also has some shortages. For example, only flows in rush hours, which are inadequate, are considered. If large amounts of traffic information, such as travel and delay times, are added into the model, then the evaluation results will be highly accurate. However, data acquisition is a long process and requires long-term cumulative observation. These topics are interesting research directions in the future.

\section{Acknowledgements}

The study was supported by the National Natural Science Foundation of China (NSFC) (grant number E080701/51508325).

This is an Open Access article distributed under the terms of the Creative Commons Attribution Licence

\section{References}

1. Wang Xinjun, Jing Dong, Su Hailong, "The Interactive Thinking of the Change of Shanghai Traffic System and the Optimization of Urban System in New Period". Journal of Urban Planning, 3, 2010, pp.35-43.

2. Zhu Hong, S.Y., Li Qinghua, "Development process and evolution trends of Jiaotong in Shanghai". Shanghai Urban Planning, 2, 2012, pp. 40-44.

3. Tong, Y., Lu, L., Zhang, Z., He, Y., \& Lu, W., "Vulnerability of Long-Distance Bridges and Tunnels in Urban Roadway Networks". Journal of Engineering Science \& Technology Review, 10(1), 2017, pp.123-130.

4. Shiqing, W., "Key Technology Study of Highway Bridge Risk Assessment". Doctoral Dissertation of Tianjin University, China, 2010, pp.56-62.

5. Sienkiewicz, J., \& Hołyst, J. A., "Statistical analysis of 22 public transport networks in Poland". Physical Review E, 72(4), 2005, pp.046127.

6. Zhang, J., Wang, S., \& Wang, X., "Comparison analysis on vulnerability of metro networks based on complex network". Physica A: Statistical Mechanics and its Applications, 496, 2018, pp.72-78.

7. Rodríguez-Núñez, E., \& García-Palomares, J. C., "Measuring the vulnerability of public transport networks". Journal of Transport Geography, 35, 2014, pp.50-63.

8. Li, X., Guo, J., Gao, C., Su, Z., Bao, D., \& Zhang, Z., "Networkbased transportation system analysis: A case study in a mountain city". Chaos, Solitons \& Fractals, 107, 2018, pp.256-265.

9. Knoop, V., \& Hoogendoorn, S., "The need of spillback simulation in assessing robustness: Concepts and a case study with dynamic route choice". In: Sixth Triennial Symposium on Transportation Analysis (Tristan), Phuket Island, Thailand: SINTEF, 2007, pp.287300 .

10. Berdica, K., "An introduction to road vulnerability: what has been done, is done and should be done". Transport Policy, 9(2), 2002, pp.117-127.

11. D'este, G. A., \& Taylor, M. A., "Network vulnerability: an approach to reliability analysis at the level of national strategic transport networks". In: Network Reliability of Transport: Proceedings of the 1st International Symposium on Transportation Network Reliability, Kyoto, Japan: INSTR, 2003, pp. 23-44.

12. Jenelius, E., \& Mattsson, L. G., "Developing a methodology for road network vulnerability analysis". Nectar Cluster, 1, 2006, pp.19 .

13. Scott, D. M., Novak, D. C., Aultman-Hall, L., \& Guo, F., "Network Robustness Index: A new method for identifying critical links and evaluating the performance of transportation networks". Journal of Transport Geography, 14(3), 2006, pp.215-227.

14. von Ferber, C., Holovatch, T., Holovatch, Y., \& Palchykov, V., "Public transport networks: empirical analysis and modeling". The European Physical Journal B, 68(2), 2009, pp.261-275.

15. Çolak, S., Luş, H., \& Atılgan, A. R., "Vulnerability of networks against critical link failures" [EB/OL]. Retrieved from https://arxiv.org/pdf/1012.5961v2.pdf, 2010-12-29/2018-12-04.
16. Kemper, A., "Valuation of network effects in software markets: a complex networks approach". Heidelberg: Physica-Verlag Heidelberg, Germang, 2009, pp.125-129.

17. Wang, X. F., Li, X., \& Chen, G. R., "Complex network theory and its application". Beijing: Tsinghua University Press, China, 2006, pp.23-26.

18. Cats, O., \& Jenelius, E., "Vulnerability analysis of public transport networks: a dynamic approach and case study for Stockholm". In: The 5th International Symposium on Transportation Network Reliability (INSTR2012), Hong Kong, China: HKSTS, 2012, pp.535-544.

19. Husdal, J., "The vulnerability of road networks in a cost-benefit perspective". In: Proceedings of the Transportation Research Board Annual Meeting (TRB 2005), Washington DC, USA: Transportation Research Board, 2005, pp.9-13.

20. Han, Y., Hu, J., Li, D., \& Zhang, S., "A novel measurement of structure properties in complex networks". In: International Conference on Complex Sciences. Shanghai, China: LNICST, 2009, pp.1292-1297.

21. Watts, D. J., \& Strogatz, S. H., "Collective dynamics of "smallworld'networks". Nature, 393(6684), 1998, pp.440.

22. Derrible, S., \& Kennedy, C., "Characterizing metro networks: state, form, and structure". Transportation, 37(2), 2010, pp.275-297.

23. Taylor, M., "Vulnerability analysis for transportation networks". Amsterdam: Elsevier, Netherlands, 2017, pp.99-103.

24. Latora, V., \& Marchiori, M., "Efficient Behavior of Small-World Networks". Physical Review Letters, 87(19), 2001, pp.198701.

25. Wang, Z., Chen, Z., Zhao, Y., \& Niu, Q., "A Novel Local Maximum Potential Point Search Algorithm for Topology Potential Field". International Journal of Hybrid Information Technology, 7(2), 2014, pp.1-8.

26. Li, M., Lu, Y., Wang, J., Wu, F. X., \& Pan, Y., “A Topology Potential-Based Method for Identifying Essential Proteins from PPI Networks". IEEE/ACM Transactions on Computational Biology \& Bioinformatics, 12(2), 2015, pp. 372.

27. Anderson, J. E., "A theoretical foundation for the gravity equation". The American Economic Review, 69(1), 1979, pp.106-116.

28. Cochrane, R., "A possible economic basis for the gravity model". Journal of Transport Economics and Policy, 9(1), 1975, pp.34-49.

29. Simini, F., González, M. C., Maritan, A., \& Barabási, A.-L., "A universal model for mobility and migration patterns". Nature, 484(7392), 2012, pp.96.

30. Mishra, S., Wang, Y., Zhu, X., Moeckel, R., \& Mahapatra, S., "Comparison between gravity and destination choice models for trip distribution in Maryland". In: Proceedings of the Transportation Research Board Annual Meeting (TRB 2013), Washington DC, USA: Transportation Research Board, 2013, pp.13-31.

31. Thiemann, C., Theis, F., Grady, D., Brune, R., \& Brockmann, D., "The structure of borders in a small world". PloS One, 5(11), 2010, pp.e15422.

32. Eusgeld, I., Kröger, W., Sansavini, G., Schläpfer, M., \& Zio, E., "The role of network theory and object-oriented modeling within a framework for the vulnerability analysis of critical infrastructures". Reliability Engineering \& System Safety, 94(5), 2009, pp.954-963. 
33. Liu, K., Wang, M., Cao, Y., Zhu, W., Wu, J., \& Yan, X., "A Comprehensive Risk Analysis of Transportation Networks Affected by Rainfall - Induced Multihazards". Risk Analysis, 38(8), 2018, pp.1618-1633.

34. Leamer, E. E., "Specification searches: Ad hoc inference with nonexperimental data". New York: John Wiley, United States, 1978, pp.156-160.

35. Raftery, A. E., "Bayesian model selection in social research". Sociological Methodology, 25, 1995, pp.111-163.

36. Doppelhofer, G., \& Miller, R. I., "Determinants of long-term growth: A Bayesian averaging of classical estimates (BACE) approach”. American Economic Review, 94(4), 2004, pp.813-835.
37. De Luca, G., \& Magnus, J. R., "Bayesian model averaging and weighted average least squares: equivariance, stability, and numerical issues". Stata Journal, 11(4), 2011, pp.518-544.

38. Magnus, J. R., Powell, O., \& Prüfer, P., "A comparison of two model averaging techniques with an application to growth empirics". Journal of Econometrics, 154(2), 2010, pp.139-153.

39. Raftery, A. E., Madigan, D., \& Hoeting, J. A., "Bayesian model averaging for linear regression models". Journal of the American Statistical Association, 92(437), 1997, pp.179-191.

40. Reyes, E. M., \& Ghosh, S. K., "Bayesian average error-based approach to sample size calculations for hypothesis testing". Journal of Biopharmaceutical Statistics, 23(3), 2013, pp.569-588. 